Research Article

\title{
Exponential Polynomials and Nonlinear Differential-Difference Equations
}

\author{
Junfeng $X u(\mathbb{D}$ and Jianxun Rong \\ Department of Mathematics, Wuyi University, Jiangmen, Guangdong 529020, China \\ Correspondence should be addressed to Junfeng Xu; xujunf@gmail.com
}

Received 5 November 2019; Revised 8 January 2020; Accepted 16 January 2020; Published 20 February 2020

Academic Editor: Ismat Beg

Copyright (C) 2020 Junfeng $\mathrm{Xu}$ and Jianxun Rong. This is an open access article distributed under the Creative Commons Attribution License, which permits unrestricted use, distribution, and reproduction in any medium, provided the original work is properly cited.

In this paper, we study finite-order entire solutions of nonlinear differential-difference equations and solve a conjecture proposed by Chen, Gao, and Zhang when the solution is an exponential polynomial. We also find that any exponential polynomial solution of a nonlinear difference equation should have special forms.

\section{Introduction and Main Result}

Extensive application of Nevanlinna theory has prompted scholars to acquire a number of results on differential equations, difference equations, and differential-difference equations. In this paper, we assume readers are familiar with the standard notations and fundamental results, see [1-4].

Given a meromorphic function $f$ and a constant $c$. We take $c=1$ for simplicity. $\Delta f(z)=f(z+1)-f(z)$ and $\Delta^{n} f(z)=\Delta\left(\Delta^{n-1} f(z)\right)$ are the first-order difference operator and $n$-th order difference operator of $f$, respectively. We adopt the notations $\rho(f)$ and $\lambda(f)$ to denote the order and the exponent of convergence of zeros of $f$, respectively.

Recall the definition of exponential polynomial of the form

$$
f(z)=P_{1}(z) e^{Q_{1}(z)}+P_{2}(z) e^{Q_{2}(z)}+\cdots+P_{k}(z) e^{Q_{k}(z)},
$$

where $P_{j}^{\prime} s$ and $Q_{j}^{\prime} s$ are polynomials in $z$. Denote

$$
\begin{aligned}
& \Gamma_{0}=\left\{e^{\alpha(z)} \mid \alpha(z) \text { is a nonconstant polynomial }\right\}, \\
& \Gamma_{1}=\left\{e^{\alpha(z)}+d \mid \alpha(z) \text { is a nonconstant polynomial and } d \in \mathbb{C}\right\}, \\
& \Gamma_{0}^{\prime}=\left\{p(z) e^{\alpha(z)} \mid p(z) \text { is a polynomial and } \alpha(z) \text { is a nonconstant polynomial }\right\} .
\end{aligned}
$$

Many papers recently have focused on solvability and existence of solutions of nonlinear differential-difference equations, see [5-16].

In 2012, Wen et al. [17] classified finite-order entire solutions of the following nonlinear difference equation:

$$
f^{n}(z)+q(z) e^{Q(z)} f(z+c)=P(z),
$$

where $n \geq 2$ is an integer and $q(z), Q(z)$, and $P(z)$ are polynomials such that $q(z)$ is not identically zero and $Q(z)$ is not a constant. They obtained the following result. 
Theorem 1 (see [17]). Let $n \geq 2$ be an integer, $c \in \mathbb{C}$, and $q(z), Q(z)$, and $P(z)$ be polynomials such that $q(z)$ is not identically zero and $\mathrm{Q}(z)$ is not a constant. Then, the finiteorder entire solutions $f$ of equation (3) should satisfy the following:

(a) Every solution $f$ satisfies $\rho(f)=\operatorname{deg} Q$ and is of mean type.

(b) Every solution $f$ satisfies $\lambda(f)=\rho(f)$ if and only if $P(z) \equiv 0$.

(c) A solution $f$ belongs to $\Gamma_{0}$ if and only if $P(z) \equiv 0$. In particular, this is the case $n \geq 3$.

(d) If a solution $f$ belongs to $\Gamma_{0}$ and $g$ is any other finiteorder entire solution to equation (3), then $f=\eta g$, where $\eta^{n-1}=1$.

(e) If $f$ is an exponential polynomial solution of form (1), then $f \in \Gamma_{1}$. Moreover, if $f \in \Gamma_{1} / \Gamma_{0}$, then $\rho(f)=1$.

In 2016, Liu [9] investigated finite-order transcendental entire solutions of the following nonlinear differential-difference equation:

$$
f^{n}(z)+q(z) e^{Q(z)} f^{(k)}(z+c)=P(z),
$$

where $n \geq 2$ and $k \geq 1$ are integers and $q(z), Q(z)$, and $P(z)$ are polynomials such that $q(z)$ is not identically zero and $Q(z)$ is not a constant. He obtained a result which is similar to Theorem 1.

In 2019, Chen et al. [6] considered solutions of equation (3), where $P(z)$ is replaced by $p_{1} e^{\lambda z}+p_{2} e^{-\lambda z}$. They obtained the following result.

Theorem 2 (see [6]). Let $n \geq 3$ be an integer and $c, \lambda, p_{1}$, and $p_{2}$ be nonzero constants. Suppose $q(z)$ and $Q(z)$ are polynomials such that $q(z)$ is nonvanishing and $Q(z)$ is not a constant. If $f$ is an entire solution of finite order of

$$
f^{n}(z)+q(z) e^{Q(z)} f(z+c)=p_{1} e^{\lambda z}+p_{2} e^{-\lambda z},
$$

then the following conclusions hold:

(1) Every solution $f$ satisfies $\rho(f)=\operatorname{deg} Q=1$.

(2) If a solution $f$ belongs to $\Gamma_{0}$, then $q(z)$ must be a constant and one of the following two relation groups holds:

(a) $f(z)=e^{(\lambda / n) z+B}$ and $Q(z)=-((n+1) / n) \lambda z+b$

(b) $f(z)=e^{-(\lambda / n)+B}$ and $Q(z)=((n+1) / n) \lambda z+b$, where both $b$ and $B$ are constants.

Remark 1. Chen et al. [6] gave an example: $f(z)=e^{z}$ is an entire solution of finite order of the following difference equation:

$$
f^{2}(z)+2 e^{-3 z} f(z-\log 2)=e^{2 z}+e^{-2 z} .
$$

From the example, they conjectured that the conclusions of Theorem 2 are still valid if $n=2$.

We consider the conjecture and prove a more generalized result. Moreover, we solve Chen, Gao, and Zhang's conjecture when $f(z)$ is an exponential polynomial of form (1).

Theorem 3. Let $k \geq 0$ be an integer and $c, \alpha_{1}, \alpha_{2}, p_{1}$, and $p_{2}$ be nonzero constants such that $\alpha_{1} \neq \alpha_{2}$. Suppose $q(z)$ is a nonvanishing polynomial and $Q(z)$ is a nonconstant polynomial. If the differential-difference equation

$$
f^{2}(z)+q(z) e^{Q(z)} f^{(k)}(z+c)=p_{1} e^{\alpha_{1} z}+p_{2} e^{\alpha_{2} z},
$$

has a transcendental entire solution $f$, then

(1) Every solution $f$ satisfies $\rho(f)=\operatorname{deg} Q \geq 1$.

(2) If $f$ is an exponential polynomial of form (1), then $\rho(f)=\operatorname{deg} Q=1$.

(3) If belongs to $\Gamma_{0}^{\prime}$, then one of the following two relation groups holds:

$$
\begin{aligned}
& \text { (a) } f(z)=g(z) e^{\left(\alpha_{2} / 2\right) z+B}, Q(z)=\left(\alpha_{1}-\left(\alpha_{2} / 2\right)\right) z+b \text {, } \\
& (g(z))^{2} e^{2 B}=p_{2} \text {, and } q(z)\left[\sum_{s=0}^{k}\left(\begin{array}{l}
k \\
s
\end{array}\right)\left(\alpha_{2} / 2\right)^{s}\right. \\
& \left.(g(z+c))^{(k-s)}\right] e^{\left(\alpha_{2} c / 2\right)+b+B}=p_{1} \\
& \text { (b) } f(z)=g(z) e^{\left(\alpha_{1} / 2\right) z+B}, Q(z)=\left(\alpha_{2}-\left(\alpha_{1} / 2\right)\right) z+b \text {, } \\
& (g(z))^{2} e^{2 B}=p_{1}, \quad \text { and } \quad q(z) \\
& {\left[\sum_{s=0}^{k}\left(\begin{array}{c}
k \\
s
\end{array}\right)\left(\alpha_{1} / 2\right)^{s}(g(z+c))^{(k-s)}\right]} \\
& e^{\left(\alpha_{1} c / 2\right)+b+B}=p_{2} \text {, where both } b \text { and } B \text { are constants } \\
& \text { and } g(z) \text { is a polynomial. }
\end{aligned}
$$

Corollary 1. Let $k \geq 0$ be an integer and $c, \alpha_{1}, \alpha_{2}, p_{1}$, and $p_{2}$ be nonzero constants such that $\alpha_{1} \neq \alpha_{2}$. Suppose $q(z)$ is a nonvanishing polynomial and $Q(z)$ is a nonconstant polynomial. If the differential-difference equation (7) has solutions fsatisfying $f \in \Gamma_{0}$, then $\rho(f)=\operatorname{deg} Q=1$ and $q(z)$ must be a constant and one of the following two relation groups holds:

(1) $f(z)=e^{\left(\alpha_{2} / 2\right) z+B}, Q(z)=\left(\alpha_{1}-\left(\alpha_{2} / 2\right)\right) z+b, e^{2 B}=p_{2}$, and $q\left(\alpha_{2} / 2\right)^{k} e^{\left(\alpha_{2} c / 2\right)+b+B}=p_{1}$

(2) $f(z)=e^{\left(\alpha_{1} / 2\right) z+B}, Q(z)=\left(\alpha_{2}-\left(\alpha_{1} / 2\right)\right) z+b, e^{2 B}=p_{1}$, and $q\left(\alpha_{1} / 2\right)^{k} e^{\left(\alpha_{2} c / 2\right)+b+B}=p_{2}$, where both $b$ and $B$ are constants.

Next, we give two examples to illustrate equation (7).

Example 1. $f(z)=e^{z+\pi i}$ is an entire solution of finite order of the following differential-difference equation:

$$
f^{2}(z)+2 e^{-3 z} f^{\prime}(z-\pi i)=2 e^{-2 z}+e^{2 z}
$$

where $k=1, \alpha_{1}=-2, \alpha_{2}=2$, and $(g(z))^{2} e^{2 B}=1=p_{2}$. Thus, case (1) occurs.

Example 2. $f(z)=2 e^{2 z}$ is an entire solution of finite order of the following difference equation:

$$
f^{2}(z)+3 e^{z-\pi i} f(z+2 \pi i)=4 e^{4 z}-6 e^{3 z},
$$

where $k=0, \alpha_{1}=4, \alpha_{2}=3$, and $(g(z))^{2} e^{2 B}=4=p_{1}$. Thus, case (2) occurs. 
In 2015, Zhang et al. [18] studied the existence of entire solutions of the following nonlinear difference equation:

$$
f^{3}(z)+q_{3}(z) \Delta^{3} f+q_{2}(z) \Delta^{2} f+q_{1}(z) \Delta f=\lambda_{1} e^{\alpha z}+\lambda_{2} e^{-\alpha z}+p(z) .
$$

They obtained the following result.

Theorem 4 (see [18]). Let $\lambda_{1}, \lambda_{2}$, and $\alpha$ be nonzero constants. Suppose $q_{j}(z)(j=1,2,3)$ and $p(z)$ are polynomials. Then, the nonlinear difference equation (10) possesses solutions of finite order of the form $f(z)=c_{1} e^{(\alpha / 3) z}+c_{2} e^{-(\alpha / 3) z}$ with $c_{1}^{3}=\lambda_{1}, c_{2}^{3}=\lambda_{2}$, and $p(z) \equiv 0 . v=e^{\alpha / 3}$ and $q_{1}, q_{2}$, and $q_{3}$ satisfy the following condition:

$$
(v-1)^{3}\left(v^{3}+1\right) q_{3}+(v-1)^{2}\left(v^{3}-v\right) q_{2}+(v-1)\left(v^{3}+v^{2}\right) q_{1}=0 .
$$

Moreover, one of the following conclusions holds:

(1) If $\alpha=(6 n \pi \pm 3 \pi) i$, then $\left(8 q_{3}-4 q_{2}+2 q_{1}\right)^{3}=27 \lambda_{1} \lambda_{2}$

(2) If $\alpha=(6 n \pi \pm \pi) i$, then $q_{1}+q_{2}=0$ and $\left( \pm \sqrt{3} i q_{1}-q_{3}\right)^{3}=27 \lambda_{1} \lambda_{2}$, where $n$ is an integer

(3) If $v \neq-1$ and $v \neq(1 \pm \sqrt{3} i) / 2$, then $q_{1}, q_{2}$, and $q_{3}$ satisfy the following equation:

$$
(v-1)^{2}\left(v^{2}-v+1\right) q_{3}+v(v-1)^{2} q_{2}+v^{2} q_{1}=0 .
$$

In the following, we consider a difference equation which is similar to (10) and obtain the following result.

Theorem 5. Suppose that $p_{1}, p_{2}$, and $\lambda$ are nonzero constants and that $a_{1}(z)$ and $a_{2}(z)$ are nonzero polynomials. If $f$ is a nontrivial exponential polynomial solution of

$$
f^{2}(z)+a_{2}(z) \Delta^{2} f+a_{1}(z) \Delta f=p_{1} e^{\lambda z}+p_{2} e^{-\lambda z},
$$

then $f$ has solutions of finite order of the following form:

$$
f(z)=c_{0}(z)+c_{1} e^{(\lambda / 2) z}+c_{2} e^{-(\lambda / 2) z},
$$

where $e^{(\lambda / 2)}= \pm i, a_{2}^{2}=-2 c_{1} c_{2}, c_{1}^{2}=p_{1}$, and $c_{2}^{2}=p_{2} . c_{0}(z)$ is a nonzero polynomial, $v=e^{(\lambda / 2)}$, and $a_{1}(z)$ and $a_{2}(z)$ satisfy

$$
\left(v^{2}-1\right)\left(a_{1} v+a_{2}(v-1)^{2}\right)=0 .
$$

Moreover, one of the following conclusions holds:

$$
\begin{aligned}
& \text { (1) If } \quad v=-1, \quad \text { then } \lambda=(4 k \pi+2 \pi) i \text {, } \\
& c_{0}(z)=a_{1}(z)-2 a_{2}(z) \neq 0, \text { and } a_{1}(z) \text { and } a_{2}(z) \\
& \text { satisfy } \\
& 2 c_{1} c_{2}+a_{2}(z)\left(a_{1}(z+2)-2 a_{2}(z+2)-a_{1}(z)+2 a_{2}(z)\right) \\
& +\left(a_{1}(z)-2 a_{2}(z)\right)\left(a_{1}(z+1)-2 a_{2}(z+1)\right)=0 \text {. }
\end{aligned}
$$

Especially, if $a_{1}$ and $a_{2}$ are constants, then $c_{0}^{2}=-2 c_{1} c_{2}$.
(2) If $v \neq-1$ and $v$ is the solution of $a_{1} v+a_{2}(v-1)^{2}=0$, then $c_{0}(z)=a_{1}(z) / 2$ and $a_{1}(z)$ and $a_{2}(z)$ satisfy

$$
a_{1}(z)^{2}-2 a_{1}(z) a_{1}(z+1)-2 a_{2}(z) \Delta^{2} a_{1}(z)=8 c_{1} c_{2} .
$$

Especially, if $a_{1}$ is a constant, then $c_{0}^{2}=-2 c_{1} c_{2}$.

The following examples show the existences of solution of equation (13).

Example 3. An entire solution $f(z)=-2+e^{\pi i z}-2 e^{-\pi i z}$ solves the following difference equation:

$$
f^{2}(z)+3 \Delta^{2} f+4 \Delta f=e^{2 \pi i z}+4 e^{-2 \pi i z},
$$

where $\quad \lambda=2 \pi i, \quad a_{1}=4, a_{2}=3, \quad c_{0}=-2 . \quad$ The case $v=\exp (\lambda / 2)=-1$ occurs.

Example 4. An entire solution $f(z)=2+e^{(\pi i / 2) z}-2 e^{-(\pi i / 2) z}$ solves the following difference equation:

$$
f^{2}(z)+2 \Delta^{2} f+4 \Delta f=e^{\pi i z}+4 e^{-\pi i z},
$$

where $\lambda=\pi i, \quad a_{1}=4, a_{2}=2, c_{0}=a_{1} / 2=2$. The case $v=e^{(\pi / 2)}=i$ satisfies $a_{1} v+a_{2}(v-1)^{2}=0$.

Example 5. An entire solution $f(z)=-2+2 e^{(\pi i / 2) z}$ $e^{-(\pi i / 2) z}$ solves the following difference equation:

$$
f^{2}(z)-2 \Delta^{2} f-4 \Delta f=4 e^{-\pi i z}+e^{\pi i z},
$$

where $\lambda=-\pi i, a_{1}=-4, a_{2}=-2, c_{0}=a_{1} / 2=-2$. The case $v=\exp (\lambda / 2)=-1$ satisfies $a_{1} v+a_{2}(v-1)^{2}=0$.

This paper is organized as follows. In Section 2, we introduce the background of exponential polynomials and some indispensable lemmas. Sections 3 and 4 contain the detailed proofs on Theorems 3 and 5. In Section 5, we will discuss the methods of the main results obtained in the paper.

\section{Preliminaries}

We recollect a basic result on exponential polynomials. Let $P(z)=b_{q} z^{q}+b_{q-1} z^{q-1}+\cdots+b_{0}$, where $b_{q} \neq 0$. We know $([1]$, p.7) that

$$
T\left(r, e^{P(z)}\right)=\left|b_{q}\right| \frac{r^{q}}{\pi}+o\left(r^{q}\right) .
$$

For exponential polynomials $f(z)$ of form (1), Wen et al. [17] followed the reasoning in [19] and acquired some instrumental tools.

Suppose the polynomials $Q_{j}(z)$ in (1) are pairwise different and normalized by $Q_{j}(0)=0$. Then, representation $(1)$ is uniquely determined and the functions $P_{j}(z) e^{Q_{j}(z)}$ are linearly independent. Let 


$$
q=\max \left\{\operatorname{deg} Q_{j} \mid Q_{j}(z) \equiv 0\right\}
$$

and let $w_{1}, w_{2}, \ldots, w_{m}$ be pairwise different leading coefficients of the polynomials $Q_{j}(z)$ of maximum degree $q$. Thus, (1) can be written in the following normalized form:

$$
f(z)=H_{0}(z)+H_{1}(z) e^{w_{1} z^{q}}+H_{2}(z) e^{w_{2} z^{q}}+\cdots+H_{m}(z) e^{w_{m} z^{q}},
$$

where $H_{i}(z)(0 \leq i \leq m)$ are either exponential polynomials of degree less than $q$ or ordinary polynomials in $z$. $H_{j}(z) \equiv 0$ hold for $1 \leq j \leq m$.

A convex hull of a set $W \subset \mathbb{C}$, denoted by $\operatorname{co}(W)$, is the intersection of all convex sets containing $W$. If $W$ contains only finitely many elements, then $\operatorname{co}(W)$ is obtained as an intersection of finitely many closed half-planes. Hence, $\operatorname{co}(W)$ is either a compact polygon (with a nonempty interior) or a line segment. We denote the perimeter of co $(W)$ by $C(\operatorname{co}(W))$. If $\operatorname{co}(W)$ is a line segment, then $C(\operatorname{co}(W))$ equals to twice the length of this line segment. We fix the notation for $W=\left\{\overline{w_{1}}, \overline{w_{2}}, \ldots, \overline{w_{m}}\right\}, W_{0}=\left\{0, \overline{w_{1}}, \ldots, \overline{w_{m}}\right\}$, and $Q(z)=b_{q} z^{q}+\cdots+b_{0}$.

Theorem 6 (see [19], Satz 1]). Let $f$ be given by (23). Then,

$$
T(r, f)=C\left(\operatorname{co}\left(W_{0}\right)\right) \frac{r^{q}}{2 \pi}+o\left(r^{q}\right)
$$

Next, we can find the following consequence from the result of Steinmetz ([20], Satz 1), i.e.,

$$
m\left(r, \frac{f^{(k)}(z+c)}{f(z)}\right)=o\left(r^{q}\right)
$$

holds for an exponential polynomial $f(z)$ in form (23) (also see [21], Section 3).

Some auxiliary results are necessary. The first one is a difference analogue of logarithmic derivative lemma given by Chiang and Feng.

Lemma 1 (see [22], Corollary 2.5). Let $f(z)$ be a meromorphic function with finite order $\rho(f)$. Suppose $c$ is a fixed nonzero complex constant. Then, for each $\varepsilon>0$, we have

$$
m\left(r, \frac{f(z+c)}{f(z)}\right)+m\left(r, \frac{f(z)}{f(z+c)}\right)=O\left(r^{\rho(f)-1+\varepsilon}\right)+O(\log r) .
$$

The following lemma is a useful tool to solve differentialdifference equations and difference equations.

Lemma 2 (see [3]). Suppose that $f_{1}(z), f_{2}(z)$, $\ldots, f_{n}(z)(n \geq 2)$ are meromorphic functions and that $g_{1}(z), g_{2}(z), \ldots, g_{n}(z)(n \geq 2)$ are entire functions. They satisfy the following conditions:

(1) $f_{1}(z) e^{g_{1}(z)}+f_{2}(z) e^{g_{2}(z)}+\cdots+f_{n}(z) e^{g_{n}(z)} \equiv 0$

(2) $g_{j}(z)-g_{k}(z)$ are not constants for $1 \leq j<k \leq n$
(3) $T\left(r, f_{j}(z)\right)=o\left(T\left(r, e^{g_{h}(z)-g_{k}(z)}\right)\right)(r \longrightarrow \infty, r \notin E)$ holds, for $1 \leq j \leq n$ and $1 \leq h<k \leq n$, where $E \subset[1, \infty)$ is finite linear measure or finite logarithmic measure.

Then, $f_{j}(z) \equiv 0(j=1,2, \ldots, n)$.

Halburd and Korhonen proved a difference analogue of Clunie lemma under the condition finite order.

Lemma 3 (see [23]). Let $f(z)$ be a nonconstant finite-order meromorphic solution of

$$
f^{n}(z) P(z, f)=Q(z, f)
$$

where $P(z, f)$ and $Q(z, f)$ are difference polynomials in $f$ with small meromorphic coefficients. Suppose $c \in \mathbb{C}$ and $\delta<1$. If the total degree of $Q(z, f)$ is a polynomial in $f$ and its shifts are less than or equal to $n$, then

$$
m(r, P(z, f))=o\left(\frac{T(r+|c|, f)}{r^{\delta}}\right)+o(T(r, f)),
$$

for all $r$ outside of a possible exceptional set with finite logarithmic measure.

Remark 2. Similar to Lemma 3 , if $f$ is a transcendental exponential polynomial in form $(23), P(z, f)$ and $Q(z, f)$ are differential-difference polynomials in $f$ and the coefficients of $P(z, f)$ and $Q(z, f)$ are polynomials $a_{i}(z)(i=1,2, \ldots, n)$, for each $\varepsilon>0$, then an obtained result is

$$
m(r, P(z, f))=o\left(r^{q}\right)
$$

where $r$ is sufficiently large.

Chen and Yang proved the following lemma.

Lemma 4 (see [24]). Let $\lambda$ be a nonzero constant and $H(z)$ be a nonvanishing polynomial. Then, the differential equation

$$
4 f^{\prime \prime}-\lambda^{2} f=H(z)
$$

has a special solution $c_{0}(z)$ which is a nonzero polynomial.

In addition, the following lemma is similar to Lemma 5.3 of [17] and Lemma 2.7 of [9]. The proof can be given word by word.

Lemma 5. Let $f$ be given by (23), where $q \geq 2$. If $f$ is a solution of equation (7), then $m=1$.

\section{Proof of Theorem 3}

Proof of Conclusion 1. Suppose that $f(z)$ is a finite-order entire solution of equation (7). Applying the lemma on the logarithmic derivative and Lemma 1 to equation (7), we obtain 


$$
\begin{aligned}
2 T(r, f(z))= & 2 m(r, f(z)) \\
= & m\left(r, p_{1} e^{\alpha_{1} z}+p_{2} e^{\alpha_{2} z}-q(z) e^{Q(z)} f^{(k)}(z+c)\right) \\
\leq & m\left(r, f(z) \frac{f(z+c)}{f(z)} \frac{f^{(k)}(z+c)}{f(z+c)}\right) \\
& +m\left(r, e^{Q(z)}\right)+O(r) \\
\leq & m(r, f(z))+m\left(r, \frac{f(z+c)}{f(z)}\right) \\
& +m\left(r, \frac{f^{(k)}(z+c)}{f(z+c)}\right)+m\left(r, e^{Q(z)}\right)+O(r) \\
\leq & T(r, f(z))+T\left(r, e^{Q(z)}\right)+O(r)+S(r, f) .
\end{aligned}
$$

Thus,

$$
T(r, f) \leq T\left(r, e^{Q}\right)+O(r)+S(r, f),
$$

which implies that $\rho(f) \leq \operatorname{deg} Q$.

If $\rho(f) \leq \operatorname{deg} Q$, then the order of left side of equation (7) is equal to $\operatorname{deg} Q$. Since the order of right side of equation (7) is equal to 1 , we have $\operatorname{deg} Q=1$ and $\rho(f)<1$. Let $Q(z)=\tilde{a} z+\widetilde{b}$, where $\tilde{a} \neq 0$. Equation (7) can be written as

$$
p_{1} e^{\alpha_{1} z}+p_{2} e^{\alpha_{2} z}+p_{3} e^{\widetilde{a} z+\widetilde{b}}+p_{4}=0,
$$

where $p_{3}=-q(z) f^{(k)}(z+c)$ and $p_{4}=-(f(z))^{2}$ satisfy $\rho\left(p_{3}\right)<1$ and $\rho\left(p_{4}\right)<1$, respectively. Next, we consider the following three cases:

Case 1. $\widetilde{a} \neq \alpha_{1}$ and $\widetilde{a} \neq \alpha_{2}$.

By equation (33) and Lemma 2, we have $p_{1}=p_{2}=0$, which is a contradiction.

Case 2. $\widetilde{a}=\alpha_{1}$ and $\widetilde{a} \neq \alpha_{2}$.

Equation (33) can be rewritten as

$$
\left(p_{1}+p_{3} e^{\tilde{b}}\right) e^{\alpha_{1} z}+p_{2} e^{\alpha_{2} z}+p_{4}=0
$$

Using Lemma 2, we have $p_{2}=0$, which is a contradiction.

Case 3. $\tilde{a} \neq \alpha_{1}$ and $\widetilde{a}=\alpha_{2}$. Similar to the proof of Case 2, we can get a contradiction.

Thus, we have $\rho(f)=\operatorname{deg} Q$. Noting $\operatorname{deg} Q \geq 1$, we obtain $\rho(f)=\operatorname{deg} Q \geq 1$.

Proof of Conclusion 2. Since $f$ is an exponential polynomial in form (1), we can consider its equivalent form (23). Suppose $q \geq 2$, by Lemma 5 we know $m=1$. That is, we have
$f(z)=H_{0}(z)+H_{1}(z) e^{w_{1} z^{q}}$. Substituting the expression of $f(z)$ into equation (7) yields

$$
\begin{aligned}
p_{1} e^{\alpha_{1} z} & +p_{2} e^{\alpha_{2} z}-\left(H_{0}(z)\right)^{2} \\
= & 2 H_{1}(z) H_{0}(z) e^{w_{1} z^{q}}+q(z) e^{Q_{0}(z)} H_{0}^{(k)}(z+c) e^{b_{q} z^{q}} \\
& +\left(H_{1}(z)\right)^{2} e^{2 w_{1} z^{q}}+q(z) e^{Q_{0}(z)+P_{1}(z)} H_{1}^{T}(z+c) e^{\left(b_{q}+w_{1}\right) z^{q}},
\end{aligned}
$$

where $Q_{0}(z)=Q(z)-b_{q} z^{q}$ and $P_{1}(z)=w_{1}(z+c)^{q}-w_{1} z^{q}$. In addition, $H_{1}^{T}(z+c)$ is a differential polynomial in $H_{1}(z+c), w_{1}(z+c)^{q}$, and their derivatives. We see that $Q_{0}(z)$ and $P_{1}(z)$ are two polynomials with degree less than or equal to $q-1$. We discuss two cases $b_{q} \neq w_{1}$ and $b_{q}=w_{1}$ :

Case 1. $b_{q} \neq w_{1}$.

Taking $b_{q}=-w_{1}, b_{q}=2 w_{1}$ and $b_{q} \notin\left\{ \pm w_{1}, 2 w_{1}\right\}$, respectively, we apply Lemma 2 to equation (35) to obtain $H_{1}(z) \equiv 0$, which is a contradiction.

Case 2. $b_{q}=w_{1}$.

Equation (35) can be rewritten as

$$
\begin{aligned}
p_{1} e^{\alpha_{1} z} & +p_{2} e^{\alpha_{2} z}-\left(H_{0}(z)\right)^{2} \\
= & {\left[2 H_{1}(z) H_{0}(z)+q(z) e^{Q_{0}(z)} H_{0}^{(k)}(z+c)\right] e^{w_{1} z^{q}} } \\
& +\left[\left(H_{1}(z)\right)^{2}+q(z) e^{Q_{0}(z)+P_{1}(z)} H_{1}^{T}(z+c)\right] e^{2 w_{1} z^{q}} .
\end{aligned}
$$

We utilize Lemma 2 again to obtain

$$
\left(H_{0}(z)\right)^{2}=p_{1} e^{\alpha_{1} z}+p_{2} e^{\alpha_{2} z} .
$$

Assume that $z_{0}$ is a zero of the above equation. Obviously, $z_{0}$ is a simple zero of $p_{1} e^{\alpha_{1} z}+p_{2} e^{\alpha_{2} z}$, but $z_{0}$ is the multiple zero of $\left(H_{0}(z)\right)^{2}$. This is a contradiction. We have $q=1 . f(z)$ is reduced to $H_{0}(z)+H_{1}(z) e^{w_{1} z}$, which implies $\rho(f)=\operatorname{deg} Q=1$.

Proof of Conclusion 3. Since $f(z)$ belongs to $\Gamma_{0}^{\prime}$, from Conclusion 2, we know that $\rho(f)=\operatorname{deg} Q=1$.

Let

$$
\begin{aligned}
& f(z)=g(z) e^{A z+B}, \\
& Q(z)=a z+b,
\end{aligned}
$$

where $a$ and $A$ are nonzero constants, $b$ and $B$ are constants, and $g(z)$ is a nonvanishing polynomial. It follows from formula (38) that

$$
f^{(k)}(z+c)=e^{A c+B}\left[\sum_{s=0}^{k}\left(\begin{array}{l}
k \\
s
\end{array}\right) A^{s}(g(z+c))^{(k-s)}\right] e^{A z} .
$$
have
Substituting formulas (38)-(40) into equation (7), we 


$$
\begin{aligned}
& \left(g(z) e^{B}\right)^{2} e^{\left(2 A-\alpha_{2}\right) z}-p_{1} e^{\left(\alpha_{1}-\alpha_{2}\right) z}-p_{2} \\
& \quad+e^{A c+b+B} q(z)\left[\sum_{s=0}^{k}\left(\begin{array}{l}
k \\
s
\end{array}\right) A^{s}(g(z+c))^{(k-s)}\right] e^{\left(a+A-\alpha_{2}\right) z}=0 .
\end{aligned}
$$

We consider the following four cases:

Case 1. $2 A-\alpha_{2}=0$ and $A+a-\alpha_{2}=0$.

Using Lemma 2, it follows from equation (41) that $p_{1}=0$. It is a contradiction.
Case 2. $2 A-\alpha_{2} \neq 0$ and $A+a-\alpha_{2} \neq 0$. If $2 A-\alpha_{2} \neq A+a-\alpha_{2} \neq \alpha_{1}-\alpha_{2}$, then we obtain $p_{1}=$ $p_{2} \equiv q(z) \equiv 0$ by equation (41) and Lemma 2. A contradiction occurs.

Now, we consider that only two of $2 A-\alpha_{2}, A+a-\alpha_{2}$, and $\alpha_{1}-\alpha_{2}$ coincide. Without loss of generality, assuming $2 A-\alpha_{2}=A+a-\alpha_{2} \neq \alpha_{1}-\alpha_{2}$, we see that equation (41) is represented as

$$
\left[\left(g(z) e^{B}\right)^{2}+e^{A c+b+B} q(z)\left[\sum_{s=0}^{k}\left(\begin{array}{l}
k \\
s
\end{array}\right) A^{s}(g(z+c))^{(k-s)}\right]\right] e^{\left(2 A-\alpha_{2}\right) z}-p_{1} e^{\left(\alpha_{1}-\alpha_{2}\right) z}-p_{2}=0 .
$$

From the above equation, using Lemma 2, we have $p_{1}=p_{2}=0$, which implies a contradiction.

Thus, $2 A-\alpha_{2}=A+a-\alpha_{2}=\alpha_{1}-\alpha_{2}$. We write equation (41) as

$$
\left[\left(g(z) e^{B}\right)^{2}+e^{A c+b+B} q(z)\left[\sum_{s=0}^{k}\left(\begin{array}{l}
k \\
s
\end{array}\right) A^{s}(g(z+c))^{(k-s)}\right]-p_{1}\right] e^{\left(\alpha_{1}-\alpha_{2}\right) z}-p_{2}=0 .
$$

We use Lemma 2 again to lead to $p_{2}=0$. It is a contradiction.

Case 3. $2 A-\alpha_{2}=0$ and $A+a-\alpha_{2} \neq 0$.

If $A+a-\alpha_{2} \neq \alpha_{1}-\alpha_{2}$, then we have $p_{1}=q(z) \equiv 0$ by equation (41) and Lemma 2. A contradiction occurs.
Thus, $A+a-\alpha_{2}=\alpha_{1}-\alpha_{2}$. We deduce $A=\left(\alpha_{2} / 2\right)$ and $a=\alpha_{1}-\left(\alpha_{2} / 2\right)$. Equation (41) can be represented as

$$
\left[q(z)\left[\sum_{s=0}^{k}\left(\begin{array}{c}
k \\
s
\end{array}\right)\left(\frac{\alpha_{2}}{2}\right)^{s}(g(z+c))^{(k-s)}\right] e^{\left(\alpha_{2} c / 2\right)+b+B}-p_{1}\right] e^{\left(\alpha_{1}-\alpha_{2}\right) z}-\left[p_{2}-\left(g(z) e^{B}\right)^{2}\right]=0 .
$$

Because of Lemma 2, we have

$$
(g(z))^{2} e^{2 B}=p_{2}
$$

$q(z)\left[\sum_{s=0}^{k}\left(\begin{array}{l}k \\ s\end{array}\right)\left(\frac{\alpha_{2}}{2}\right)^{s}(g(z+c))^{(k-s)}\right] e^{\left(\alpha_{2} c / 2\right)+b+B}=p_{1}$
We proceed to obtain $f(z)=g(z) e^{\left(\alpha_{2} / 2\right) z+B}$ and $\mathrm{Q}(z)=\left(\alpha_{1}-\left(\alpha_{2} / 2\right)\right) z+b$.

Case 4. $2 A-\alpha_{2} \neq 0$ and $A+a-\alpha_{2}=0$.

If $2 A-\alpha_{2} \neq \alpha_{1}-\alpha_{2}$, then we obtain $p_{1}=0$ by equation (41) and Lemma 2. A contradiction occurs.

Thus, $2 A-\alpha_{2}=\alpha_{1}-\alpha_{2}$. We derive $A=\left(\alpha_{1} / 2\right)$ and $a=\alpha_{2}-\left(\alpha_{1} / 2\right)$. Equation (41) is equivalent to 


$$
\left[\left(g(z) e^{B}\right)^{2}-p_{1}\right] e^{\left(\alpha_{1}-\alpha_{2}\right) z}-\left[p_{2}-q(z)\left[\sum_{s=0}^{k}\left(\begin{array}{c}
k \\
s
\end{array}\right)\left(\frac{\alpha_{2}}{2}\right)^{s}(g(z+c))^{(k-s)}\right] e^{\left(\alpha_{1} c / 2\right)+b+B}\right]=0 .
$$

By Lemma 2, we have

$$
\begin{aligned}
(g(z))^{2} e^{2 B} & =p_{1} \\
q(z)\left[\sum_{s=0}^{k}\left(\begin{array}{c}
k \\
s
\end{array}\right)\left(\frac{\alpha_{2}}{2}\right)^{s}(g(z+c))^{(k-s)}\right] e^{\left(\alpha_{1} c / 2\right)+b+B} & =p_{2} .
\end{aligned}
$$

Consequently, we obtain $f(z)=g(z) e^{\left(\alpha_{1} / 2\right) z+B}$ and $Q(z)=\left(\alpha_{2}-\left(\alpha_{1} / 2\right)\right) z+b$.

\section{Proof of Theorem 5}

Assume that the difference equation (13) has a transcendental entire solution $f$ of finite order.

Applying Lemma 1 to equation (13), we have

$$
\begin{aligned}
T\left(r, p_{1} e^{\lambda z}+p_{2} e^{-\lambda z}\right)= & T\left(r, f^{2}(z)+a_{2}(z) \Delta^{2} f+a_{1}(z) \Delta f\right) \\
\leq & T\left(r, f^{2}\right)+T\left(r, a_{2} \Delta^{2} f+a_{1} \Delta f\right)+O(1) \\
\leq & T\left(r, f^{2}\right)+m\left(r, \frac{a_{2} \Delta^{2} f+a_{1} \Delta f}{f}\right) \\
& +m(r, f) \\
\leq & T\left(r, f^{2}\right)+m(r, f)+S(r, f) \\
\leq & 3 T(r, f)+S(r, f) .
\end{aligned}
$$

On the other hand, we deduce

$$
\begin{aligned}
T\left(r, p_{1} e^{\lambda z}+p_{2} e^{-\lambda z}\right)= & T\left(r, f^{2}+a_{2}(z) \Delta^{2} f+a_{1}(z) \Delta f\right) \\
\geq & T\left(r, f^{2}\right)-T\left(r, a_{2}(z) \Delta^{2} f+a_{1}(z) \Delta f\right)+O(1) \\
\geq & 2 T(r, f)-m\left(r, \frac{a_{2} \Delta^{2} f+a_{1} \Delta f}{f}\right) \\
& -m(r, f)+O(1) \\
\geq & 2 T(r, f)-T(r, f)+S(r, f) \\
= & T(r, f)+S(r, f) .
\end{aligned}
$$

Combining equations (48) and (49), it follows that $T(r, f)+S(r, f) \leq T\left(r, p_{1} e^{\lambda z}+p_{2} e^{-\lambda z}\right) \leq 3 T(r, f)+S(r, f)$,

which implies $\rho(f)=1$.

Denoting $P_{1}(f):=a_{2}(z) \Delta^{2} f+a_{1}(z) \Delta f$, we rewrite equation (13) as

$$
f^{2}+P_{1}(f)=p_{1} e^{\lambda z}+p_{2} e^{-\lambda z} .
$$

Differentiating equation (51) twice on both sides, we have

$$
\begin{aligned}
2 f f^{\prime}+P_{1}^{\prime}(f) & =\lambda\left(p_{1} e^{\lambda z}-p_{2} e^{-\lambda z}\right), \\
2\left(f^{\prime}\right)^{2}+2 f f^{\prime \prime}+P_{1}^{\prime \prime}(f) & =\lambda^{2}\left(p_{1} e^{\lambda z}+p_{2} e^{-\lambda z}\right) .
\end{aligned}
$$

By equations (51) and (53), we obtain

$$
\left(f^{\prime}\right)^{2}=\frac{1}{2} \lambda^{2} f^{2}-f f^{\prime \prime}+Q_{1}(f)
$$

where $Q_{1}(f)=(1 / 2)\left[\lambda^{2} P_{1}(f)-P_{1}^{\prime \prime}(f)\right]$. Eliminating $e^{\lambda z}$ and $e^{-\lambda z}$ from equations (51) and (52), we have

$$
\lambda^{2}\left[f^{2}+P_{1}(f)\right]^{2}-\left[2 f f^{\prime}+P_{1}^{\prime}(f)\right]^{2}=4 p_{1} p_{2} \lambda^{2}
$$

which implies

$$
\lambda^{2} f^{4}-4 f^{2}\left(f^{\prime}\right)^{2}=R_{3}(f),
$$

where

$$
\begin{aligned}
R_{3}(f)= & 4 f f^{\prime} P_{1}^{\prime}(f)+\left[P_{1}^{\prime}(f)\right]^{2}+4 p_{1} p_{2} \lambda^{2}-2 \lambda^{2} f^{2} P_{1}(f) \\
& -\lambda^{2}\left[P_{1}(f)\right]^{2} .
\end{aligned}
$$

Substituting equation (54) into equation (56) yields

$$
f^{3}\left(4 f^{\prime \prime}-\lambda^{2} f\right)=T_{3}(f)
$$

where $T_{3}(f)=4 f^{2} Q_{1}(f)+R_{3}(f)$ is a differential-difference polynomial in $f$ and its total degree is not greater than three.

Now, we discuss two cases.

Case 1. $T_{3}(f) \equiv 0$.

It follows from equation (58) that

$$
4 f^{\prime \prime}-\lambda^{2} f \equiv 0
$$

The general entire solution $f(z)$ of the above equation is

$$
f(z)=c_{1} e^{(\lambda / 2) z}+c_{2} e^{-(\lambda / 2) z}
$$

where $c_{1}$ and $c_{2}$ are constants satisfying $c_{1} c_{2} \equiv 0$. We obtain 


$$
\begin{gathered}
\Delta f(z)=c_{1}\left(e^{(\lambda / 2)}-1\right) e^{(\lambda / 2) z}+c_{2}\left(e^{-(\lambda / 2)}-1\right) e^{-(\lambda / 2) z} \\
\Delta^{2} f(z)=c_{1}\left(e^{(\lambda / 2)}-1\right)^{2} e^{(\lambda / 2) z}+c_{2}\left(e^{-(\lambda / 2)}-1\right)^{2} e^{-(\lambda / 2) z} .
\end{gathered}
$$

Substituting formulas (60)-(62) into equation (13) yields

$$
\begin{aligned}
& 2 c_{1} c_{2}+\left(c_{1}^{2}-p_{1}\right) e^{\lambda z}+\left(c_{2}^{2}-p_{2}\right) e^{-\lambda z} \\
& \quad+c_{1}\left[a_{1}(z)\left(e^{(\lambda / 2)}-1\right)+a_{2}(z)\left(e^{(\lambda / 2)}-1\right)^{2}\right] e^{(\lambda / 2) z} \\
& \quad+c_{2}\left[a_{1}(z)\left(e^{-(\lambda / 2)}-1\right)+a_{2}(z)\left(e^{-(\lambda / 2)}-1\right)^{2}\right] e^{-(\lambda / 2) z}=0 .
\end{aligned}
$$

By Lemma 2 and equation (63), we deduce $c_{1} c_{2} \equiv 0$, which is a contradiction.

Case 2. $T_{3}(f) \equiv 0$.

Noting that $f$ is an exponential polynomial in (23) with the order 1 , we have

$f(z)=H_{0}(z)+H_{1}(z) e^{w_{1} z}+H_{2}(z) e^{w_{2} z}+\cdots+H_{m}(z) e^{w_{m} z}$,

where $H_{0}(z), H_{1}(z), \ldots, H_{m}(z)$ are polynomials. Therefore,

$$
\begin{aligned}
& 4 f^{\prime \prime}(z)-\lambda^{2} f(z)=\left(4 H_{0}^{\prime \prime}(z)-\lambda^{2} H_{0}(z)\right)+\sum_{i=1}^{m}\left(4 H_{i}^{\prime \prime}(z)\right. \\
& \left.+4 w_{1} H_{i}^{\prime}(z)+4 H_{i}^{\prime}(z)+4 w_{1} H_{i}(z)-\lambda^{2} H_{i}(z)\right) .
\end{aligned}
$$

Since equation (58) satisfies conditions of Lemma 3 and Remark 2, it follows that

$$
m\left(r, 4 f^{\prime \prime}-\lambda^{2} f\right)=o(r)
$$

From this, (65), and Theorem 6, we know that that $4 f^{\prime \prime}-\lambda^{2} f$ is a polynomial. By equation (58) and $T_{3}(f) \equiv 0$, we have

$$
4 f^{\prime \prime}-\lambda^{2} f=H(z)
$$

where $H(z)$ is a nonvanishing polynomial. By Lemma 4 , the above equation has a nonzero polynomial solution $c_{0}(z)$. Then, the general entire solution $f(z)$ of $4 f^{\prime \prime}-\lambda^{2} f=H(z)$ can be represented as

$$
f(z)=c_{0}(z)+c_{1} e^{(\lambda / 2) z}+c_{2} e^{-(\lambda / 2) z},
$$

where $c_{0}(z)$ is nonzero polynomial and $c_{1}$ and $c_{2}$ are constants. It is easy to verify

$$
\begin{aligned}
\Delta f(z)= & {\left[c_{0}(z+1)-c_{0}(z)\right]+c_{1}\left(e^{(\lambda / 2)}-1\right) e^{(\lambda / 2) z} } \\
& +c_{2}\left(e^{-(\lambda / 2)}-1\right) e^{-(\lambda / 2) z} \\
\Delta^{2} f(z)= & {\left[c_{0}(z+2)-2 c_{0}(z+1)+c_{0}(z)\right] } \\
& +c_{1}\left(e^{(\lambda / 2)}-1\right)^{2} e^{(\lambda / 2) z}+c_{2}\left(e^{-(\lambda / 2)}-1\right)^{2} e^{-(\lambda / 2) z}
\end{aligned}
$$

Substituting formulas (68) and (70) into equation (13) yields

$$
\begin{aligned}
& \left(c_{1}^{2}-p_{1}\right) e^{\lambda z}+\left(c_{2}^{2}-p_{2}\right) e^{-\lambda z} \\
& +\left[2 c_{1} c_{0}(z)+a_{1}(z) c_{1}\left(e^{(\lambda / 2)}-1\right)+a_{2}(z) c_{1}\left(e^{(\lambda / 2)}-1\right)^{2}\right] e^{(\lambda / 2) z} \\
& +\left[2 c_{2} c_{0}(z)+a_{1}(z) c_{2}\left(e^{-(\lambda / 2)}-1\right)+a_{2}(z) c_{2}\left(e^{-(\lambda / 2)}-1\right)^{2}\right] e^{-(\lambda / 2) z} \\
& +\left[2 c_{1} c_{2}+\left(c_{0}(z)\right)^{2}+a_{1}(z) c_{0}(z+1)-a_{1}(z) c_{0}(z)\right. \\
& \left.\quad+a_{2}(z) c_{0}(z+2)-2 a_{2}(z) c_{0}(z+1)+a_{2}(z) c_{0}(z)\right]=0 .
\end{aligned}
$$

By Lemma 2 and equation (70), we deduce

$$
\begin{gathered}
c_{1}^{2}=p_{1} \neq 0, \\
c_{2}^{2}=p_{2} \neq 0, \\
2 c_{0}(z)+a_{1}(z)\left(e^{(\lambda / 2)}-1\right)+a_{2}(z)\left(e^{(\lambda / 2)}-1\right)^{2} \equiv 0, \\
2 c_{0}(z)+a_{1}(z)\left(e^{-(\lambda / 2)}-1\right)+a_{2}(z)\left(e^{-(\lambda / 2)}-1\right)^{2} \equiv 0, \\
2 c_{1} c_{2}+\left(c_{0}(z)\right)^{2}+a_{1}(z) c_{0}(z+1)-a_{1}(z) c_{0}(z) \\
+a_{2}(z) c_{0}(z+2)-2 a_{2}(z) c_{0}(z+1)+a_{2}(z) c_{0}(z) \equiv 0 .
\end{gathered}
$$

From (72) and (73), we have $a_{1}\left(e^{(\lambda / 2)}-e^{-(\lambda / 2)}\right)+a_{2}\left(e^{(\lambda / 2)}-e^{-(\lambda / 2)}\right)\left(e^{(\lambda / 2)}+e^{-(\lambda / 2)}-2\right)=0$,

Set $v=e^{(\lambda / 2)}$, it follows that

$$
\left(v^{2}-1\right)\left(a_{1} v+a_{2}(v-1)^{2}\right)=0 .
$$

If $v=1$, then $\lambda=4 k \pi i$, and substituting $v=1$ into (72) or (73), we obtain $c_{0}(z) \equiv 0$. It is a contradiction. 
If $v=-1$, then $\lambda=(4 k \pi+2 \pi) i$, and substituting $v=-1$ into (72) or (73), we obtain $c_{0}(z)=a_{1}(z)-2 a_{2}(z) \neq 0$ and (74) can be reduced to

$$
\begin{aligned}
2 c_{1} c_{2}+ & a_{2}(z)\left(a_{1}(z+2)-2 a_{2}(z+2)-a_{1}(z)+2 a_{2}(z)\right) \\
& +\left(a_{1}(z)-2 a_{2}(z)\right)\left(a_{1}(z+1)-2 a_{2}(z+1)\right)=0 .
\end{aligned}
$$

Especially, if $a_{1}$ and $a_{2}$ are constants, then $c_{0}^{2}=-2 c_{1} c_{2}$. If $v \neq \pm 1$ and $v$ is the solution of $a_{1} v+a_{2}(v-1)^{2}=0$. From (72) or (73), we have $c_{0}(z)=a_{1}(z) / 2$. Equation (74) can be reduced to

$$
a_{1}(z)^{2}-2 a_{1}(z) a_{1}(z+1)-2 a_{2}(z) \Delta^{2} a_{1}(z)=8 c_{1} c_{2} .
$$

Especially, if $a_{1}$ is a constants, then $c_{0}^{2}=-2 c_{1} c_{2}$.

\section{Conclusions}

In this study, we mainly consider the solution of two equations when the solution is an exponential polynomial.

First, we consider the nonlinear differential-difference equation (7) proposed by Chen et al. [6]. They conjecture that the conclusions of Theorem 2 are still valid. We consider the conjecture in Theorem 3. In the first step, we proved that $\rho(f)=\operatorname{deg} Q$. From this, it seems plausible that $f$ is an exponential polynomial of form (1). In the second step, we confirmed that $\rho(f)=\operatorname{deg} Q=1$ when $f$ is an exponential polynomial. In the last step, we give the solution when $f$ belongs to $\Gamma_{0}^{\prime}$ by Conclusion 2 .

Second, we consider a difference equation which is similar to (10), where $f^{3}(z)$ is also replaced by $f^{2}(z)$. Since we cannot prove that $4 f^{\prime \prime}-\lambda^{2} f$ is a polynomial if $f$ has no restriction, a new Clunie Lemma is given in Remark 2 where $f$ is an exponential polynomial. We obtain the expression of the solution of equation (13) if the solution is an exponential polynomial by the special Clunie Lemma.

\section{Data Availability}

The data used to support the findings of this study are available from the corresponding author upon request.

\section{Conflicts of Interest}

The authors declare that they have no conflicts of interest.

\section{Acknowledgments}

This research was supported by the National Natural Science Foundation of China (no. 11871379), Natural Science Foundation of Guangdong Province (no. 2018A0303130058), and Funds of Education Department of Guangdong (2016KTSCX145).

\section{References}

[1] W. K. Hayman, Meromorphic Function, Clarendon Press, Oxford, UK, 1964.

[2] I. Laine, Nevanlinna Thoery and Complex Differential Equations, Walter de Gruyter, Berlin, Germany, 1993.
[3] C. C. Yang and H. X. Yi, Uniqueness Theory of Meromorphic Functions, Springer, Dordrecht, Netherlands, 2003.

[4] L. Yang, Value Distribution Theory, Springer-Verlag, Berlin, Germany, 1993.

[5] M. F. Chen and Z. S. Gao, "Entire solutions of certain type of nonlinear differential equations and differential-difference equations," Journal of Computational Analysis and Applications, vol. 24, pp. 137-147, 2018.

[6] M.-F. Chen, Z.-S. Gao, and J.-L. Zhang, "Entire solutions of certain type of non-linear difference equations," Computational Methods and Function Theory, vol. 19, no. 1, pp. 17-36, 2019.

[7] C. P. Li, F. Lü, and J. F. Xu, "Entire solutions of nonlinear differential-difference equations," SpringerPlus, vol. 5, no. 1, p. 609, 2016.

[8] Z. Latreuch, "On the existence of entire solutions of certain class of non-linear difference equations," Mediterranean Journal of Mathematics, vol. 14, no. 3, pp. 1-16, 2017.

[9] K. Liu, "Exponential polynomials as solutions of differentialdifference equations of certain types," Mediterranean Journal of Mathematics, vol. 13, no. 5, pp. 3015-3027, 2016.

[10] F. Lü and Q. Han, "On the Fermat-type equation $f^{3}(z)+f^{3}(z+c)=1$," Aequationes Mathematicae, vol. 91, no. 1, pp. 129-136, 2017.

[11] F. Lü, W. R. Lü, C. P. Li, and J. Xu, "Growth and uniqueness related to complex differential and difference equations," Results in Mathematics, vol. 74, no. 1, p. 30, 2019.

[12] X. Qi, N. Li, and L. Yang, "Uniqueness of meromorphic functions concerning their differences and solutions of difference Painlevé equations," Computational Methods and Function Theory, vol. 18, no. 4, pp. 567-582, 2018.

[13] X. G. Qi and L. Z. Yang, "A note on meromorphic solutions of complex differential-difference equations," Bulletin of the Korean Mathematical Society, vol. 56, no. 3, pp. 597-607, 2019.

[14] J. Rong and J. Xu, "Three results on the nonlinear differential equations and differential-difference equations," Mathematics, vol. 7, no. 6, p. 539, 2019.

[15] N. Xu, T. B. Cao, and K. Liu, "Entire solutions for nonlinear differential-difference equations," Electronic Journal of Differential Equations, vol. 22, pp. 1-8, 2015.

[16] C.-C. Yang and I. Laine, "On analogies between nonlinear difference and differential equations," Proceedings of the Japan Academy, Series A, Mathematical Sciences, vol. 86, no. 1, pp. 10-14, 2010.

[17] Z. T. Wen, J. Heittokangas, and I. Lain, "Exponential polynomials as solutions of certain nonlinear difference equations," Acta Mathematica Sinica, English Series, vol. 28, no. 7, pp. 1295-1306, 2012.

[18] F. R. Zhang, N. N. Liu, W. R. Lü, and C. Yang, "Entire solutions of certain class of differential-difference equations," Advances in Difference Equations, vol. 2015, no. 1, Article ID 150, 2015.

[19] N. Steinmetz, "Zur Wertverteilung von exponentialpolynomen," Manuscripta Mathematica, vol. 26, no. 1-2, pp. 155$167,1978$.

[20] N. Steinmetz, "Zur Wertverteilung der quotienten von exponentialpolynomen," Archiv der Mathematik, vol. 35, no. 1, pp. 461-470, 1980.

[21] Z.-T. Wen, G. G. Gundersen, and J. Heittokangas, "Dual exponential polynomials and linear differential equations," Journal of Differential Equations, vol. 264, no. 1, pp. 98-114, 2018. 
[22] Y.-M. Chiang and S.-J. Feng, "On the Nevanlinna characteristic of $f(z+\eta)$ and difference equations in the complex plane," The Ramanujan Journal, vol. 16, no. 1, pp. 105-129, 2008.

[23] R. G. Halburd and R. J. Korhonen, "Difference analogue of the lemma on the logarithmic derivative with applications to difference equations," Journal of Mathematical Analysis and Applications, vol. 314, no. 2, pp. 477-487, 2006.

[24] Z.-X. Chen and C.-C. Yang, "On entire solutions of certain type of differential-difference equations," Taiwanese Journal of Mathematics, vol. 18, no. 3, pp. 677-685, 2014. 